\title{
A universal form of internal displacement field based quadrilateral area coordinate method QACM-III
}

\author{
Chen Xiaoming ${ }^{1,}$, , Duan Jin and Li Yungui \\ ${ }^{1}$ China state construction technical center, Beijing, China \\ aHanee@126.com
}

\begin{abstract}
Keywords: Finite element; Quadrilateral area coordinate; Generalized conforming; Internal displacement field

Abstract. By supplement appropriate internal displacement field on the nodal displacement of the element, the complementary of displacement polynomial can be increased, so the accuracy of element can be improved and the element may be more insensitive to mesh distortion. But in order to keep compatibility of source element, internal displacement field should be researched according to different source elements. In this paper, based on generalized conforming theory, the third quadrilateral area coordinate method QACM-III was used to develop universal form for plane elements. This universal internal displacement field can be used easily for those plane elements formulated with QACM-III.
\end{abstract}

\section{Introduction}

Isoparametric coordinate method was used widely for developing quadrilateral elements for its convenience in formulation especially for those conforming elements. By using isoparametric coordinate, compatible elements can be developed much more easily than using Cartesian coordinate. But it has been proved that those conforming elements may exhibit over stiffness because of excessive constraints on element sides which are used to ensure convergence. For improving the performance of conforming elements, in 1973, professor Wilson[1] developed the famous incompatible element Q6 by supplement internal displacement field for Q4 element. Compared with Q4 element, new element Q6 can satisfy quadratic complementary about isoparametric coordinate, so it exhibits more accurate in simulating the beams under pure bending. But its internal displacement field only can keep conforming at element nodes, so Q6 can not present exact solution of patch test for irregular quadrangles. In 1976, Taylor[2] modified the internal displacement field of Q6 by using numerical integral method and developed element QM6, which can pass the patch test and still keep high accuracy. Although it is successful in formulation of QM6, but method of numerical integration may be difficult to be used for other incompatible elements.

Based on generalized conforming theory, a universal form of additional internal displacement field was developed by using isoparametric coordinate method[3]. This universal form can be used easily for improving the performance of quadrilateral plane elements.

Because of nonlinear relationship between isoparametric coordinate and Cartesian coordinate [4], the complementary of displacement polynomial would descend rapidly when the elements degenerated to irregular quadrangles, and then accuracy would degraded seriously, Long et al. [5,6] developed the first kind of quadrilateral area coordinate QACM-I to overcome this problem. After this, the second kind and the third kind of quadrilateral area coordinate named QACM-II[7] and QACM-III[8] were developed as the supplement of QACM-I. Those elements formulated with quadrilateral area coordinates exhibit good accuracy and insensitivity to mesh distortion $[9,10]$.

Most of these new elements formulated with quadrilateral area coordinate methods can present exact solution for patch test, but some of them are the same as Q6 element, Chen et al. [11] researched different methods to make them pass the patch test. In this paper, a universal form of internal displacement field was formulated by using QACM-III based on generalized conforming theory. This universal form can be used easily for those elements formulated with QACM-III, and it would improve the property without changing the compatibility of source elements. 


\section{Introduction of QACM-III}

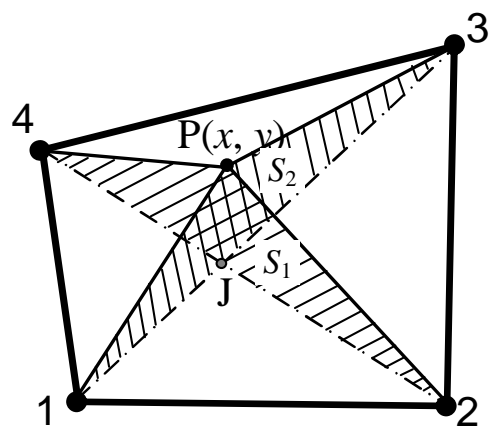

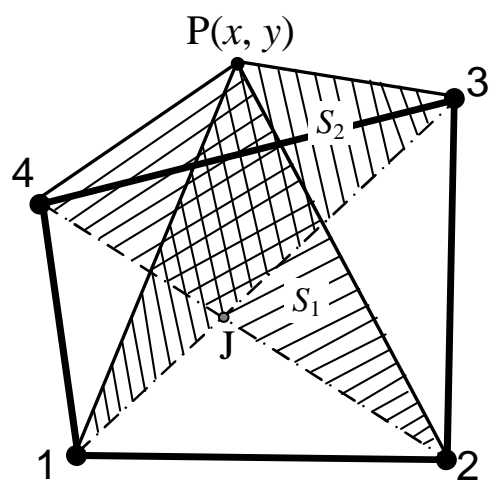

Figure 1. Definition of the quadrilateral area coordinates $T_{i}$ of the OACM-III

The two-component area coordinates are specified by $\mathrm{T} 1$ and $\mathrm{T} 2$, which are defined as

$$
T_{1}=\frac{S_{1}}{A}, T_{2}=\frac{S_{2}}{A}
$$

Where $A$ is the area of the element; $S_{1}$ and $S_{2}$ are the generalized areas of $\Delta \mathrm{P} 42$ and $\Delta \mathrm{P} 13$.

The relationship between QACM-III and Cartesian coordinates is as follows:

$$
\left\{\begin{array}{l}
T_{1} \\
T_{2}
\end{array}\right\}=\frac{1}{2 A}\left[\begin{array}{cc}
y_{4}-y_{2} & x_{4}-x_{2} \\
y_{1}-y_{3} & x_{3}-x_{1}
\end{array}\right]\left\{\begin{array}{l}
x \\
y
\end{array}\right\}
$$

And the relationship between QACM-III and isoparametric coordinates is as follows:

$$
\left\{\begin{array}{l}
T_{1}=\frac{1}{4}\left[\xi+\eta+\left(g_{3}-g_{1}\right)(1+\xi \eta)\right] \\
T_{2}=\frac{1}{4}\left[-\xi+\eta+\left(g_{4}-g_{2}\right)(1-\xi \eta)\right]
\end{array}\right.
$$

The transformations of the derivatives of the first order can be written as

$$
\left\{\begin{array}{c}
\frac{\partial}{\partial x} \\
\frac{\partial}{\partial y}
\end{array}\right\}=\frac{1}{2 A}\left[\begin{array}{ll}
y_{4}-y_{2} & y_{1}-y_{3} \\
x_{2}-x_{4} & x_{3}-x_{1}
\end{array}\right]\left\{\begin{array}{c}
\frac{\partial}{\partial T_{1}} \\
\frac{\partial}{\partial T_{2}}
\end{array}\right\}
$$

The area integral formulae for the first, second terms are as follows

$$
\begin{gathered}
\iint_{A}\left\{\begin{array}{l}
T_{1} \\
T_{2}
\end{array}\right\} \mathrm{d} A=\frac{A}{3}\left\{\begin{array}{l}
g_{3}-g_{1} \\
g_{4}-g_{2}
\end{array}\right\} \\
\iint_{A}\left\{\begin{array}{c}
T_{1}^{2} \\
T_{2}^{2} \\
T_{1} T_{2}
\end{array}\right\} \mathrm{d} A=\frac{A}{24}\left\{\begin{array}{c}
1+3\left(g_{3}-g_{1}\right)^{2} \\
1+3\left(g_{4}-g_{2}\right)^{2} \\
2\left(g_{3}-g_{1}\right)\left(g_{4}-g_{2}\right)
\end{array}\right\}
\end{gathered}
$$

\section{Internal displacement field of QACM-III}

The universal form of internal displacement field in QACM-III can be assumed as:

$$
\bar{u}=T_{1}^{n} T_{2}^{m}+\lambda_{1} T_{1}+\lambda_{2} T_{2}+\lambda_{0}
$$

Where $\lambda_{0}, \lambda_{1}, \lambda_{2}$ are undetermined parameters, and $n, m$ are arbitrary natural numbers.

In order to pass the patch test, the incompatible energy at element sides induced by internal displacement filed should be zero, then the conforming conditions that internal displacement field should be satisfied are as follows. 


$$
\begin{aligned}
& \iint \frac{\partial \bar{u}}{\partial x} d A=\sqrt{N^{\prime}} \bar{u} d s=0 \\
& \iint \frac{\partial \bar{u}}{\partial y} d A=\tilde{N}^{\prime}{ }^{\prime} \bar{u} d s=0 \\
& \iint \bar{u} d A=0
\end{aligned}
$$

Where $l^{\prime}$ and $m$ are the direction cosine of element sides.

Substitute the internal displacement field of Eq.7 into Eq.8, then:

$$
\begin{aligned}
\iint \frac{\partial \bar{u}}{\partial x} d A & =\iint\left(\frac{y_{4}-y_{2}}{2 A} \frac{\partial \bar{u}}{\partial T_{1}}+\frac{y_{1}-y_{3}}{2 A} \frac{\partial \bar{u}}{\partial T_{2}}\right) d A \\
& =\iint\left(\frac{y_{4}-y_{2}}{2 A}\left(n T_{1}^{n-1} T_{2}^{m}+\lambda_{1}\right)+\frac{y_{1}-y_{3}}{2 A}\left(m T_{1}^{n} T_{2}^{m-1}+\lambda_{2}\right)\right) d A \\
& =\frac{n\left(y_{4}-y_{2}\right)}{2 A} \iint T_{1}^{n-1} T_{2}^{m} d A+\frac{m\left(y_{1}-y_{3}\right)}{2 A} \iint T_{1}^{n} T_{2}^{m-1} d A+\frac{\left(y_{4}-y_{2}\right)}{2} \lambda_{1}+\frac{\left(y_{1}-y_{3}\right)}{2} \lambda_{2} \\
& =0
\end{aligned}
$$

And:

$$
\iint \frac{\partial \bar{u}}{\partial y} d A=\frac{n\left(x_{2}-x_{4}\right)}{2 A} \iint T_{1}^{n-1} T_{2}^{m} d A+\frac{m\left(x_{3}-x_{1}\right)}{2 A} \iint T_{1}^{n} T_{2}^{m-1} d A+\frac{x_{2}-x_{4}}{2} \lambda_{1}+\frac{x_{3}-x_{1}}{2} \lambda_{2}=0
$$

Then $\lambda_{1}, \lambda_{2}$ can be written as:

With

$$
\left\{\begin{array}{l}
\lambda_{1} \\
\lambda_{2}
\end{array}\right\}=-\frac{1}{A}\left\{\begin{array}{c}
n \iint T_{1}^{n-1} T_{2}^{m} d A \\
m \iint T_{1}^{n} T_{2}^{m-1} d A
\end{array}\right\}
$$

$$
\iint \bar{u} d A=\iint T_{1}^{n} T_{2}^{m} d A-\frac{n}{A} \iint T_{1}^{n-1} T_{2}^{m} d A \iint T_{1} d A-\frac{m}{A} \iint T_{1}^{n} T_{2}^{m-1} d A \iint T_{2} d A+\lambda_{0} A=0
$$

The other undetermined parameter can be derived out as:

$$
\lambda_{0}=-\frac{1}{A} \iint T_{1}^{n} T_{2}^{m} d A+\frac{n}{A^{2}} \iint T_{1}^{n-1} T_{2}^{m} d A \iint T_{1} d A+\frac{m}{A^{2}} \iint T_{1}^{n} T_{2}^{m-1} d A \iint T_{2} d A
$$

Let $I_{n, m}^{T}=\iint T_{1}^{n} T_{2}^{m} d A$, then:

$$
\lambda_{0}=-\frac{1}{A} I_{n, m}^{T}+\frac{n}{A^{2}} I_{n-1, m}^{T} I_{1,0}^{T}+\frac{m}{A^{2}} I_{n, m-1}^{T} I_{0,1}^{T}
$$

The universal form of internal displacement of QACM-III can be simplified as follows:

$$
\bar{u}=T_{1}^{n} T_{2}^{m}-\frac{n}{A} I_{n-1, m}^{T} T_{1}-\frac{m}{A} I_{n, m-1}^{T} T_{2}+\lambda_{0}
$$

Then any polynomial which can satisfy the patch test can be formulated with Eq.17. Some special terms of internal displacement field are presented as follows.

For quadratic polynomials:

$$
\begin{aligned}
n=2, m & =0 \\
\bar{u} & =T_{1}^{2}+\lambda_{1} T_{1}+\lambda_{0} \\
\lambda_{1} & =-\frac{2}{A} \iint T_{1} d A=-\frac{2}{3}\left(g_{3}-g_{1}\right) \\
\lambda_{0} & =-\frac{1}{A} \iint T_{1}^{2} d A+\frac{2}{A^{2}} \iint T_{1} d A \iint T_{1} d A=-\frac{1}{24}\left[1+3\left(g_{3}-g_{1}\right)^{2}\right]+\frac{2}{9}\left(g_{3}-g_{1}\right)^{2} \\
n=1, m=1 &
\end{aligned}
$$




$$
\begin{aligned}
\bar{u} & =T_{1} T_{2}+\lambda_{1} T_{1}+\lambda_{2} T_{2}+\lambda_{0} \\
\lambda_{1} & =-\frac{1}{A} \iint T_{2} d A=-\frac{1}{3}\left(g_{4}-g_{2}\right) \\
\lambda_{2} & =-\frac{1}{A} \iint T_{1} d A=-\frac{1}{3}\left(g_{3}-g_{1}\right) \\
\lambda_{0} & =-\frac{1}{A} \iint T_{1} T_{2} d A+\frac{2}{A^{2}} \iint T_{1} d A \iint T_{2} d A=\frac{5}{36}\left(g_{3}-g_{1}\right)\left(g_{4}-g_{2}\right) \\
n=0, m= & 2 \\
\bar{u} & =T_{2}^{2}+\lambda_{2} T_{2}+\lambda_{0} \\
\lambda_{2} & =-\frac{2}{A} \iint T_{2} d A=-\frac{2}{3}\left(g_{4}-g_{2}\right) \\
\lambda_{0} & =-\frac{1}{A} \iint T_{2}^{2} d A+\frac{2}{A^{2}} \iint T_{2} d A \iint T_{2} d A=-\frac{1}{24}\left[1+3\left(g_{4}-g_{2}\right)^{2}\right]+\frac{2}{9}\left(g_{4}-g_{2}\right)^{2}
\end{aligned}
$$

For cubic polynomials.

$n=3, m=0$

$$
\begin{aligned}
\bar{u} & =T_{1}^{3}+\lambda_{1} T_{1}+\lambda_{0} \\
\lambda_{1} & =-\frac{3}{A} \iint T_{1}^{2} d A=-\frac{1}{8}\left[1+3\left(g_{3}-g_{1}\right)^{2}\right] \\
\lambda_{0} & =-\frac{1}{A} \iint T_{1}^{3} d A+\frac{3}{A^{2}} \iint T_{1}^{2} d A \iint T_{1} d A=-\frac{1}{120}\left(g_{3}-g_{1}\right)\left[1+3\left(g_{3}-g_{1}\right)^{2}\right] \\
n=2, m=1 & \\
\bar{u} & =T_{1}^{2} T_{2}+\lambda_{1} T_{1}+\lambda_{2} T_{2}+\lambda_{0} \\
\lambda_{1} & =-\frac{2}{A} \iint T_{1} T_{2} d A=-\frac{1}{6}\left(g_{3}-g_{1}\right)\left(g_{4}-g_{2}\right) \\
\lambda_{2} & =-\frac{1}{A} \iint T_{1}^{2} d A=-\frac{1}{24}\left[1+3\left(g_{3}-g_{1}\right)^{2}\right] \\
\lambda_{0} & =-\frac{1}{A} \iint T_{1}^{2} T_{2} d A+\frac{2}{A^{2}} \iint T_{1} T_{2} d A \iint T_{1} d A+\frac{1}{A^{2}} \iint T_{1}^{2} d A \iint T_{2} d A \\
& =-\frac{1}{180}\left(g_{4}-g_{2}\right)\left[1+3\left(g_{3}-g_{1}\right)^{2}\right]+\frac{1}{18}\left(g_{4}-g_{2}\right)\left(g_{3}-g_{1}\right)^{2} \\
n=1, m=2 & \\
\bar{u} & =T_{1} T_{2}^{2}+\lambda_{1} T_{1}+\lambda_{2} T_{2}+\lambda_{0} \\
\lambda_{1} & =-\frac{1}{A} \iint T_{2}^{2} d A=-\frac{1}{24}\left[1+3\left(g_{4}-g_{2}\right)^{2}\right] \\
\lambda_{2} & =-\frac{2}{A} \iint T_{1} T_{2} d A=-\frac{1}{6}\left(g_{3}-g_{1}\right)\left(g_{4}-g_{2}\right) \\
\lambda_{0} & =-\frac{1}{A} \iint T_{1} T_{2}^{2} d A+\frac{1}{A^{2}} \iint T_{2}^{2} d A \iint T_{1} d A+\frac{2}{A^{2}} \iint T_{1} T_{2} d A \iint T_{2} d A \\
& =\frac{1}{180}\left(g_{3}-g_{1}\right)\left[1+3\left(g_{4}-g_{2}\right)^{2}\right]+\frac{1}{18}\left(g_{3}-g_{1}\right)\left(g_{4}-g_{2}\right)^{2} \\
n=0, m & =-14
\end{aligned}
$$




$$
\begin{aligned}
& \bar{u}=T_{2}^{3}+\lambda_{2} T_{2}+\lambda_{0} \\
& \lambda_{2}=-\frac{3}{A} \iint T_{2}^{2} d A=-\frac{1}{8}\left[1+3\left(g_{4}-g_{2}\right)^{2}\right] \\
& \lambda_{0}=-\frac{1}{A} \iint T_{2}^{3} d A+\frac{3}{A^{2}} \iint T_{2}^{2} d A \iint T_{2} d A=-\frac{1}{120}\left(g_{4}-g_{2}\right)\left[1+3\left(g_{4}-g_{2}\right)^{2}\right]
\end{aligned}
$$

These polynomials can be used as the internal displacement field for those plane elements formulated with QACM-III, and if the nodal displacement can satisfy the conforming condition, the new element would pass the strict form of patch test.

\section{Conclusions}

Proper internal displacement field can increase the complementary of polynomial for finite elements, then the accuracy and convergence would be increased significantly, and the elements would be more insensitive to mesh distortion. In this paper, universal polynomial forms of quadrilateral area coordinate QACM-III are formulated for those plane elements developed with the same coordinate method. Quadratic and cubic polynomials are presented as examples for other researchers.

\section{References}

[1] E L Wilson, R L Taylor, et al., Incompatible displacement models, Numerical and Computer Methods in Structural Mechanics, In: S J Fenves et al., eds, Academic Press, New York, 1973, 43-57.

[2] R L Taylor, P J Beresford and E L Wilson, A non-conforming element for stress analysis, Int.J.Num.Meth.Engng., 1976(10): 1211-1219.

[3] Zhang Chunsheng, Long Yuqiu, Xu Yin. Three-dimensional basic formulations of additional incompatible displacement of internal parameters. Engineering Mechanics. 2011, 18(5): 50-63.

[4] N S Lee, K J Bathe. Effects of element distortion on the performance of isoparametric elements. Int. J. Num. Meth. Engng. 1993(36): 3553-3576.

[5] Y.Q. Long, J.X. Li, Z.F. Long, and S. Cen. Area coordinates used in quadrilateral elements. Communications in Numerical Methods in Engineering, vol. 15, no. 8, pp. 533-545, 1999.

[6] Z.F. Long, J.X. Li, S. Cen, and Y.Q. Long. Some basic formulae for Area coordinates used in quadrilateral elements. Communications in Numerical Methods in Engineering, vol. 15, no. 12, pp. 841-852, 1999.

[7] X.M. Chen, S. Cen, X.R. Fu, and Y.Q. Long, "A new quadrilateral area coordinate method (QACM-II) for developing quadrilateral finite element models," International Journal for Numerical Methods in Engineering, vol. 73, no. 13, pp. 1911-1941, 2008.

[8] Z.F. Long, S. Cen, L. Wang, X.R. Fu, and Y.Q. Long, "The third form of the quadrilateral area coordinate method (QACM-III): Theory, application, and scheme of composite coordinate interpolation," Finite Elements in Analysis and Design, vol. 46, no. 10, pp. 805-818, 2010.

[9] Xiaoming Chen, Song Cen, Yuqiu Long et al. Membrane elements insensitive to distortion using the quadrilateral area coordinate method. Computers \& Structures, 2004, 82(1): 35-54.

[10] A.K. Soh, Long Zhifei and Cen Song. Development of a new quadrilateral thin plate element using area coordinates. Computer Methods in Applied Mechanics and Engineering, 2000, 190(8-10): 979 987

[11] Chen Xiaoming,Cen Song, Li Yungui, Sun JianYun. Several treatments on non-conforming element failed in the strict patch test. Mathematical Problems in Engineering, vol. 2013, Article ID 901495. doi:10.1155/2013/901495. 